\title{
Marfan syndrome
}

\author{
Eesha Jain, Ramesh Kumar Pandey
}

Department of Paediatric and Preventive Dentistry, Faculty of Dental Sciences, King George's Medical University, Lucknow, Uttar Pradesh, India

\section{Correspondence to}

Dr Eesha Jain,

drej.84@gmail.com

\section{CrossMark}

To cite: Jain E, Pandey RK. BMJ Case Rep Published

online: [please include Day

Month Year] doi:10.1136/

bcr-2013-201632

\section{SUMMARY}

Marfan syndrome is a rare autosomal dominant disorder of the connective tissue, with skeletal, ligamentous, orooculofacial, pulmonary, abdominal, neurological and the most fatal, cardiovascular manifestations. It has no cure but early diagnosis, regular monitoring and preventive lifestyle regimen ensure a good prognosis. However, the diagnosis can be difficult as it is essentially a clinical one, relying on family history, meticulous physical examination and investigation of involved organ systems. Patients of Marfan syndrome portray very typical physical and orofacial characteristics, suggesting obvious recognition, but due to variable phenotypic expression, cases often go unnoticed unless a full range of attributing features is apparent. Dental practitioners are very likely to encounter patients of Marfan syndrome at an early age as they frequently present for dental treatment. The present case report illustrates the preliminary screening of Marfan syndrome in a dental office followed by timely diagnosis and appropriate referrals.

\section{BACKGROUND}

Type I or classic Marfan syndrome involves mutation in FBN1 gene, located on chromosome 15, which encodes for microfibrillar glycoprotein fibrillin $1 .{ }^{12}$ Any decrease in the quantity or quality of fibrillin mars the resiliency of the connective tissue to normal tissue stress. The condition affects the skeleton, lungs, eyes, heart and aorta. The affected individuals portray as tall, slender, with funnel shaped chest, having long, thin extremities and unusually long, spidery digits, joint instability and scoliosis, suggesting obvious recognition, but due to varying degrees of expressivity and penetrance, cases often go unnoticed unless a full gamut of attributing features is apparent. ${ }^{3}$ Patients often remain oblivious of underlying systemic defects and may suddenly be faced by serious life-threatening sequelae in adulthood, the most fatal ones being cardiovascular in nature. ${ }^{4}$ Dilation of the aortic root can lead to congestive heart failure while aortic aneurysms can undergo rupture, which is the most common cause of death in patients of Marfan syndrome. ${ }^{5}$

Surveillance of involved body organs is very crucial to keep morbidity at bay, underscoring the importance of an early and correct diagnosis. With the lack of specific laboratory tools, the mainstay of identification rests with the Ghent criteria, delineating major and minor diagnostic features of the syndrome. ${ }^{6}$

It is critical to highlight here the role of the dentist in screening such patients as the need for dental treatment is often their first foray into the healthcare system. Dental practitioners must be well informed of the orofacial as well as physical attributes in Marfan syndrome as this can enable them to make a preliminary diagnosis of the patients and make timely referrals to cardiologists, ophthalmologists, orthopaedic surgeons, genetists and psychologists for comprehensive management.

\section{CASE PRESENTATION}

A 13-year-old girl presented with a painful tooth in the upper left back region. Her medical history revealed that she suffered from patent ductus arteriosus which was surgically closed when she was 6 years. The patient gave a history of shortness of breath and dyspnoea which was resolved following the intervention. She reported of frequent sore throat, cough, middle ear infections and difficulty hearing. The patient also had a malformation of the right tragus for which she was operated on 4 years ago. Her medical records noted that she suffered from brachial arch syndrome and she also had malformation of the right sternocleidomastoid, which restricted her neck movement. The girl was born full term and her family history was unremarkable.

The patient appeared too thin and slender with her body weight measuring less than average for her age and sex, hinting at an aesthenic body type. She had disproportionately long arms and legs as compared with the trunk and her arm span was more than her height by about 3 inches with an increased floor to pubis measurement/pubis to vertex measurement (figure 1A). Examination of her hands showed elongated fingers with thickening at the phalange joints (figure 1B). The girl presented with positive Walker Murdoch wrist sign, that is, overlapping of the complete distal phalanx and little finger when wrapped around the opposite wrist and a positive Steinberg thumb sign, that is, extension of the distal phalanx of the thumb beyond the ulnar border of the hand when apposed across the palm (figure 1C) indicating extreme ligamentous laxity (figure 1D). An examination of the feet revealed that she had flat feet with mild pronation along with elongated toes. Another finding was a subtly indented chest and mild hunching of the back.

Extraoral examination revealed a very narrow cranium with dolicocephaly and leptoprosopic facial features. Also evident were malar hypoplasia, mandibular retrognathia, macrostomia and downward slanting palpebral fissures (figure 2A). Intraoral findings included a highly crowded dentition with anterior and posterior crossbites, higharched palate and Angle's class II molar relation on both sides (figure 2B,C). The girl had poor-oral 
Figure 1 The patient showing (A) arachnodactyly, (B) dolichostenomelia and (C and D) ligamentous laxity.

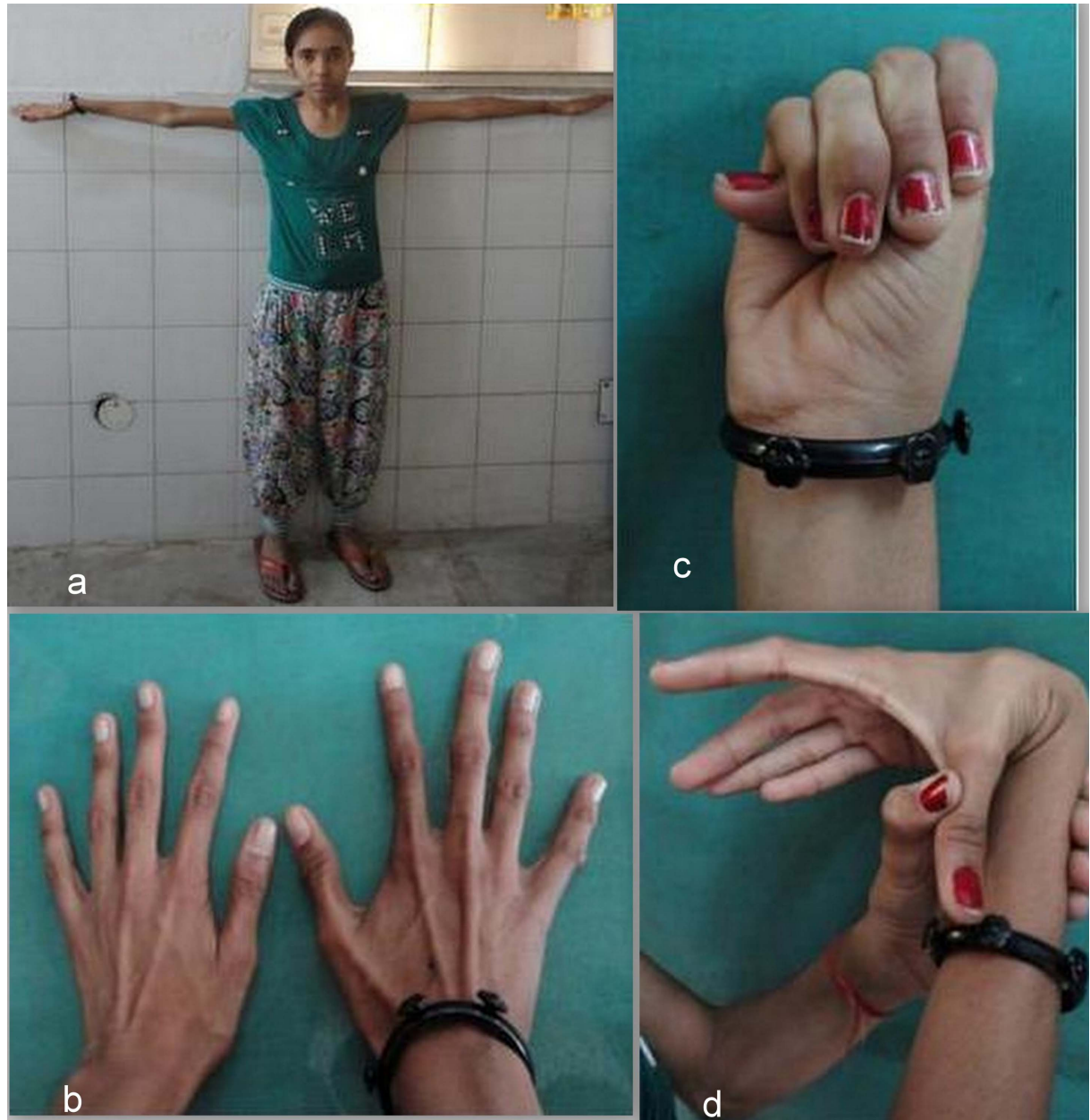

d hygiene and anterior marginal gingivitis. She also reported of pain on chewing although there was no tenderness or clicking in the temporomandibular joint (TMP). The patient had an abscess in relation to a grossly carious deciduous maxillary left first molar.

\section{INVESTIGATIONS}

The patient's orthopantomogram showed a lobulated radiolucency in relation to permanent maxillary left premolars and first molar, which was suspected to be a radicular cyst or a septate maxillary sinus (figure 3). However, a dental CT scan of the region ruled out
Figure 2 Facial and intraoral photographs of the patient showing (A) dolicocephaly and leptoprosopic facial form and (B and C) crowding and a high arched narrow palate.

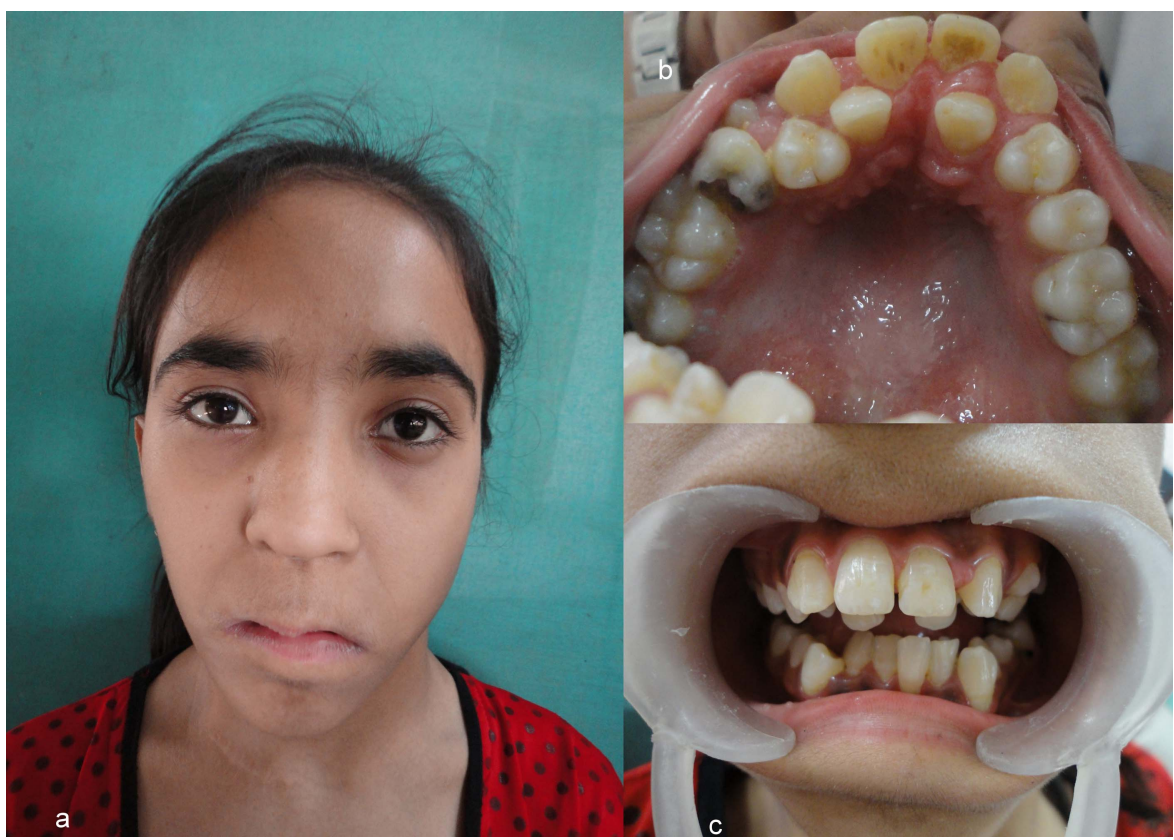


any such possibility and was simply a developmental anomaly with the floor of the left antrum dipping between the roots of teeth. Otorhinolaryngological examination revealed a deviated nasal septum but no sinus pathology was found.

\section{DIFFERENTIAL DIAGNOSIS}

Homocystinuria clinically resembles Marfan syndrome. Ectopia lentis and pectus excavatum/carinatum are common to both conditions. However, homocystinuria also presents with malar flush, osteoporosis and mental retardation but no dissecting aneuryms which are characteristic of Marfan syndrome. Homocystinuria can be diagnosed by a positive urinary nitroprusside test.

\section{TREATMENT}

Oral prophylaxis was performed for the patient and her grossly carious deciduous maxillary left first molar was extracted under local anaesthesia. All these procedures were performed following a $50 \mathrm{mg} / \mathrm{kg}$ body weight amoxicillin prophylactic dose taken $1 \mathrm{~h}$ prior, owing to the patient's cardiac pathology. Subsequently, the patient was referred to the department of orthodontics for further evaluation and correction of malocclusion. The orthodontic treatment in such patients can be carried out as in healthy patients but with challenges such as taking utmost care to maintain the health of the periodontium and administering prophylactic antibiotics before any invasive procedures. The treatment objective in this case would be to attain an Angle's class II molar relation and a class I canine relation following the bilateral extraction of the maxillary premolars. The crowding is subsequently resolved utilising the spaces created by the alignment of teeth using fixed mechanotherapy, which corrects rotated teeth and also helps to achieve a proper over jet and corrects lip incompetency. The curve of spee is levelled by the controlled intrusion of the upper and lower anterior teeth which also corrects the overbite. Maxillary expansion is performed to correct a bilateral posterior cross bite using a succession of nickel titanium and stainless steel wires or by using the Hyrax appliance. Maxillary expansion also improves nasal patency and compensates for nasal airway resistance and possible obstructive sleep apnoea. The corrected occlusion is necessarily followed by a retention phase employing usage of Hawley's appliance or lingually bonded retainers.

\section{OUTCOME AND FOLLOW-UP}

The patient had an uneventful healing following the extraction and was scheduled for a 6-month follow-up to evaluate the oral hygiene status.

\section{DISCUSSION}

Marfan syndrome is a condition which not only has systemic manifestations but also mars the appearance and quality of life of the affected. The compromised physical capacity and cosmetic issues limit the life opportunities for the patients as well as leads to frustration and low self-esteem. Constriction of the maxilla, crowded dentition and concomitant cross-bites typify the oral manifestations for which patients seek aesthetic correction. ${ }^{7}$ Such cases also show high-arched palate associated with narrow nasal airway and compensatory mouth breathing which alters natural head position leading to the development of adenoid facies. High nasal airway resistance also increases the susceptibility to obstructive sleep apnoea. ${ }^{8}$

Furthermore, connective tissue disorders are associated with a periodontal bone loss too. ${ }^{9}$ Although there is lack of an established correlation between Marfan syndrome and severe periodontitis, reports of alveolar bone loss, high attachment level loss, furcation involvement and tooth hypermobility in affected individuals suggest some consonance between the two. ${ }^{10}$ Temporomandibular defects may be present owing to articular deformation and ligament hyperlaxity, evident in the form of TMJ subluxation, clicking and pain during mastication. ${ }^{11}$ Bauss et $a l^{12}$ have also reported of regressive alterations of teeth such as pulpoliths and calcific obliterations in patients of Marfan syndrome, which may be faced during endodontic therapies.

Another key consideration is that cardiac pathology in these patients increases the risk of endocarditis following any dental treatment that might result in bleeding. Thus, it is incumbent to perform any invasive dental procedure following an antibiotic prophylaxis. ${ }^{9}$ If the patient requires extensive dental treatment, it may be advisable to resort to sedation or general anaesthesia to avoid medicating the patient multiple times. The need of oral hygiene maintenance cannot be emphasised enough in such patients as frequent scaling increases the potential for bacteraemia. Hence, the patients must be motivated for adequate home preventive measures for good oral hygiene which include powered and adapted toothbrushes, topical fluoride application and anti bacterial mouthwashes. Hirota $e a^{13}$ suggest caution while selecting an anaesthetic as epinephrine can produce an
Figure 3 Dentascan showing grossly carious left deciduous, maxillary first molar and septate left maxillary sinus.

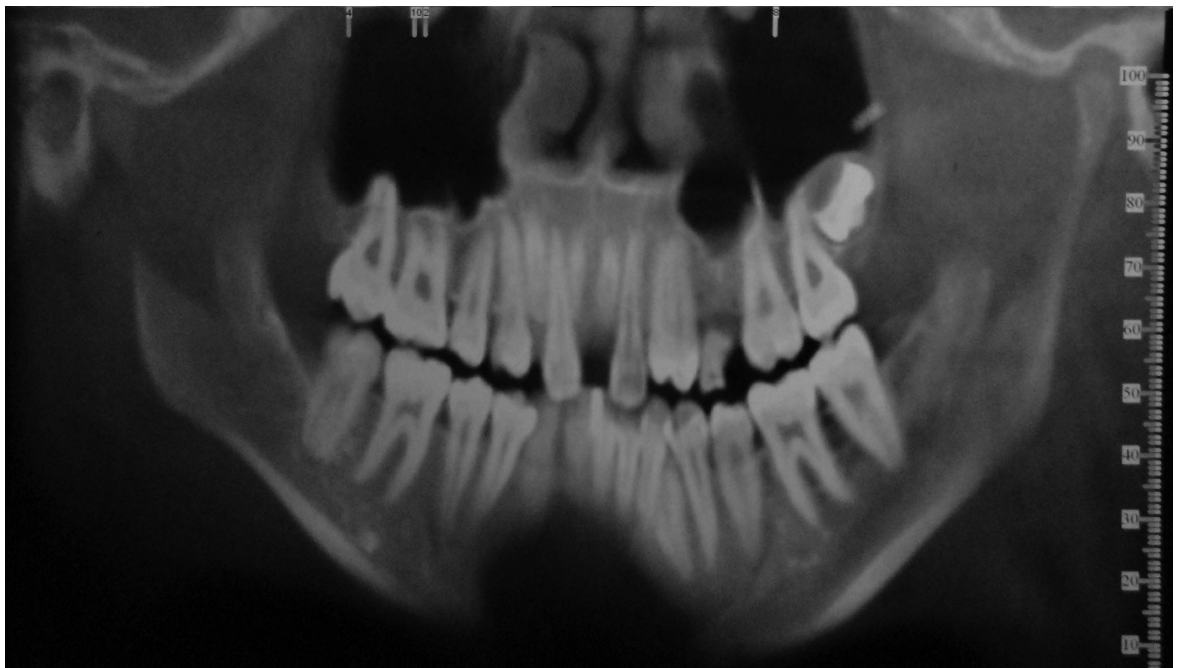


accelerated cardiac function. Orthognathic surgeries, if required, can be performed under regular monitoring by the cardiologist.

The dentist's role further extends in counselling the patient to avoid strenuous exercise and contact sports to prevent deleterious effects of increased blood pressure on the diseased aorta. Lower intensity dynamic exercises are recommended instead of high-intensity isometric ones. ${ }^{14}$ Patients should be advised annual ophthalmic check-ups as they are at an increased risk of glaucoma, cataract and retinal detachment. Besides, early correction of high-refractive error in childhood can preserve maximum visual function. Dramatic progression of skeletal deformities during the growth periods also necessitates regular orthopaedic evaluation. Most importantly, assessment of cardiac functions and status of aortic root by regular echocardiograms can be a life-saving measure. Smoking is contraindicated as it can lead to emphysema. As extreme air pressure changes can cause pneumothorax, flying in unpressurised chambers and deep sea diving can be hazardous as well. Dentists should also be well versed with the pharmacological means to delay aortic dilation which includes $\beta$-blockers, ACE inhibitors and calcium channel blockers.

Ting et al reported that facial features are more specific than sensitive for the presence of Marfan syndrome. Though

\section{Learning points}

- Progressive and potentially fatal complications of Marfan syndrome warrant an early diagnosis.

- Diagnosis mainly rests on a clinical correlation of physical attributes, family history and detailed multiorgan evaluation.

- Dental practitioners must be well informed of the orofacial as well as physical attributes in Marfan syndrome as they are very liable to encounter affected patients in their office at an early age.

- Marfan syndrome should always be considered as a diagnostic possibility when the patient presents as exceptionally tall, lean, with long spidery digits and limbs, with long narrow face and crowded dentition.

- Early diagnosis leading to institution of lifestyle modifications, preventive regimen and regular monitoring of involved organ systems holds the key to increased life expectancy in patients of Marfan syndrome. orofacial features cannot be relied on as a sole screening tool for making an initial diagnosis yet they can certainly be used to prioritise patients for appropriate referrals and detailed examination. ${ }^{15}$ The early recognition of Marfan syndrome also aids lifestyle and career guidance for the patient along with emotional counselling besides providing an opportunity for family screening and genetic evaluation of the condition.

Competing interests None.

Patient consent Obtained

Provenance and peer review Not commissioned; externally peer reviewed.

\section{REFERENCES}

1 Pyeritz RE. Toward understanding cause and pathogenesis of Marfan syndrome. In: Hetzer R, Gehle P, Ennker J, eds. Cardiovascular aspects of Marfan syndrome. Darmstadt, Germany: Steinkopff Verlag, 1995:1-8.

2 von Kodolitsch Y, Robinson PN. Marfan syndrome: an update of genetics, medical and surgical management. Heart 2007:93:755-60.

3 Pyeritz RE, Gasner C. The Marfan syndrome. 4th edn. New York: The National Marfan Foundation, 1994.

4 Allwork SP, Miall-Allen VM, Wyse RK, et al. Cardiovascular disease in Marfan patients in infancy and childhood. In: Hetzer R, Gehle P, Ennker J, eds. Cardiovascular aspects of Marfan syndrome. Darmstadt, Germany: Steinkopff Verlag, 1995:17-23.

5 Robiscek F, Thubrikar M. The mechanism and prevention of aortic dissection in Marfan syndrome. In: Hetzer R, Gehle P, Ennker J, eds. Cardiovascular aspects of Marfan syndrome. Darmstadt, Germany: Steinkopff Verlag, 1995:61-70.

6 Ades L; CSANZ Cardiovascular Genetics Working Group. Guidelines for the diagnosis and management of Marfan syndrome. Heart Lung Circ 2007:16:28-30.

7 De Coster PJ, Martens LC, De Paepe A. Orofacial manifestations of congenital fibrillin deficiency: pathogenesis and clinical diagnostics. Pediatr Dent 2004:26:535-7.

8 Cistulli PA, Sullivan CE. Influence of maxillary morphology on nasal airway resistance in Marfan's syndrome. Acta Otolaryngol 2000;120:410-13.

9 Nualart Grollmus ZC, Morales Chavez MC, Silvestre Donat FJ. Periodontal disease associated to systemic genetic disorders. Med Oral Patol Oral Cir Bucal 2007;12: E211-15

10 Straub AM, Grahame R, Scully C, et al. Severe periodontitis in Marfan's syndrome: a case report. J Periodontol 2002;73:823-6.

11 Bauss 0 , Sadat-Khonsari R, Fenske C, et al. Temporomandibular joint dysfunction in Marfan syndrome. Oral Surg Oral Med Oral Pathol Oral Radiol Endod 2004:97:592-8.

12 Bauss O, Neter D, Rahman A. Prevalence of pulp calcifications in patients with Marfan syndrome. Oral Surg Oral Med Oral Pathol Oral Radiol Endod 2008;106: e56-61.

13 Hirota $Y$, Sugiyama K, Niwa H, et al. Systemic management of Marfan's syndrome during dental treatment: a case report. Anesth Pain Control Dent 1993:2:162-70.

14 Braverman AC. Exercise and the Marfan syndrome. Med Sci Spots Exerc 1998:30:387-95

15 Ting BL, Dietz HC III, Deepti M, et al. Sponseller the diagnostic value of the facial features of Marfan syndrome. J Child Orthop 2010;4:545-51.

Copyright 2013 BMJ Publishing Group. All rights reserved. For permission to reuse any of this content visit http://group.bmj.com/group/rights-licensing/permissions.

BMJ Case Report Fellows may re-use this article for personal use and teaching without any further permission.

Become a Fellow of BMJ Case Reports today and you can:

- Submit as many cases as you like

- Enjoy fast sympathetic peer review and rapid publication of accepted articles

- Access all the published articles

- Re-use any of the published material for personal use and teaching without further permission

For information on Institutional Fellowships contact consortiasales@bmjgroup.com

Visit casereports.bmj.com for more articles like this and to become a Fellow 